\title{
EFEITOS DA REESTRUTURAÇÃO PRÓ-INOVAÇÃO NOS CUSTOS EM SAÚDE
}

\section{EFFECTS OF INNOVATIVE ORGANIZATIONAL STRUCTURES ON HEALTH COSTS}

Recebido em 08.04.2014. Aprovado em 27.10.2015

Avaliado pelo sistema double blind review

Cristina Monken Avellar

DOI: http://dx.doi.org/10.12712/rpca.v9i4.570

cristina.avellar@ini.fiocruz.br

Instituto Nacional de Infectologia Evandro Chagas (INI), Rio de Janeiro/RJ, BRASIL

\section{Marcelino José Jorge}

marcelino.jorge@ini.fiocruz.br

Instituto Nacional de Infectologia Evandro Chagas (INI), Rio de Janeiro/RJ, BRASIL

\section{Frederico Azevedo de Carvalho}

fdecarv@gmail.com

Universidade Federal do Rio de Janeiro (UFRJ), Rio de Janeiro/RJ, BRASIL

\section{Rui Américo Mathiasi Horta}

rui.horta@ufjf.edu.br

Universidade Federal de Juiz de Fora (UFJF), Juiz de Fora/MG, BRASIL

\section{Resumo}

O objetivo do artigo é avaliar o efeito da configuração Organização Inovadora no desempenho em custo da pesquisa clínica associada à assistência em saúde. O método é um estudo de caso sobre evolução da pesquisa e dos custos do Laboratório de Pesquisa Clínica em DST e AIDS do Instituto Nacional de Infectologia Evandro Chagas da Fundação Oswaldo Cruz (Lapclin-AIDS/INI/Fiocruz), com base em estimativas do Projeto de Avaliação e Acompanhamento - Indicadores Gerencias do INI para 2006 e 2009, e no uso do teste de Spearman para analisar a correlação entre despesas e custos unitários da atenção clínica no INI. O resultado é que a nova estrutura organizacional não minimiza custos em sentido estrito, mas promove a pesquisa e a distribuição eficiente dos recursos entre laboratórios. A conclusão é que essa abordagem permite medir o desempenho em custo face à mudança organizacional e confirma a efetividade em custo da reestruturação da pesquisa do INI segundo o Modelo de Atenção Integral em Saúde.

Palavras-chave: Pesquisa Clínica em HIV e AIDS. Estrutura organizacional. Microcustos. Custeio Baseado em Atividades. Análise de desempenho em custos.

\section{Abstract}

The aim of this paper is to assess the effect of innovative organizational structures on the performance in costs of clinical research associated with health care. The method is a case study about research and costs of the Clinical Research Laboratory on STD and AIDS of the Evandro Chagas National Institute of Infectious Diseases, Oswaldo Cruz Foundation (Lapclin-AIDS/INI/Fiocruz). Uses estimates for 2006 and 2009 of the INI's project about Assessment and Monitoring - Management Indicators, and the Spearman test to analyze correlation between annual expenses and unit costs of INI's health procedures. The result is that the new organizational structure does not strictly minimize costs, but promotes research and efficient distribution of resources between labs. The conclusion is that this approach allows to measure performance in cost due to organizational change and confirms the effectiveness on costs of the restructuration of INI's research under the Comprehensive Health Care Model.

Keywords: Clinical Research on HIV and AIDS. Organizational structure. Microcosts. Activity Based Costing. Costs' performance analysis. 


\section{Introdução}

No decorrer das últimas décadas, as mudanças no processo de trabalho e na estrutura organizacional foram significativas: a organização rígida ehierarquizada foi substituída pelo trabalho em equipe, enquanto as estruturas de divisão de trabalho e coordenação foram remodeladas, de forma a promover a atitude empreendedora e o maior aproveitamento dos ganhos associados à inovação.

Em consequência, o ambiente em que as organizações produtivas estão inseridas apresenta-se cada vez mais complexo e a demanda por informações mais acuradas para auxiliar a tomada de decisão é cada vez maior (SOUZA et al., 2009). Essa transformação exige mais que capacidade organizacional para se adaptar às novas estruturas: "é preciso desenvolver uma capacidade contínua de adaptação e mudança" (ROBBINS, 1999: 407).

A reestruturação da Fundação Oswaldo Cruz (Fiocruz) na década de 1990, por exemplo, tendo incorporado alguns dos princípios da doutrina de administração pública gerencial, resultou na adoção do modelo de gestão orientada para resultados para a promoção da pesquisa "estratégica" em saúde, a pesquisa orientada para missões (COZZENS; MELKERS, 1997).

Nesse sentido, o Instituto Nacional de Infectologia Evandro Chagas (INI), uma Unidade TécnicoCientífica (UTC) da Fiocruz, cuja missão é contribuir para a melhoria das condições de saúde da população brasileira no campo das doenças infecciosas, adotou a estrutura organizacional Ações Integradas de pesquisa clínica, desenvolvimento tecnológico, ensino e atenção clínica de referência em doenças infecciosas (PAI) em 1999, com vistas a obter ganhos de eficiência aliado aos de efetividade no tratamento dessas nosologias.

Uma vez que a distribuição do orçamento da Administração Central da Fiocruz (AC) entre as UTC é regulada pelo seu plano estratégico - Plano Anual (PA), o monitoramento das despesas e custos do INI recorre a estimativas do seu Projeto de Avaliação e Acompanhamento - Indicadores Gerenciais (PAAIGs), que são obtidas com base na aplicação dos princípios do método Custeio Baseado em Atividades, tendo em vista que as transferências de recursos do orçamento da AC para custear as despesas do INI, por este motivo denominadas Despesas Indiretas no Projeto PAA-IGs, são substanciais.
Por outro lado, a diversidade de laboratórios de pesquisa clínica do INI, dedicados a um amplo espectro de doenças infecciosas coberto pelas PAI, e das suas atividades de atenção clínica, ensino e pesquisa, bem como a natureza multiprofissional da atenção clínica que prestam, resultam na necessidade de levantamento, em nível de microcustos, das despesas com as atividades dos laboratórios inscritas no orçamento do INI (denominadas Despesas Diretas pelo PAA-IGs, por estarem sob o controle da gestão do INI).

No contexto do desdobramento posterior da reestruturação organizacional da Fiocruz, ao final de 2006 foram aprovadas as conclusões de estudos recomendando a adoção do modelo Organização Inovadora - ou configuração Adhocrática apud Mintzberg (1995) - nas UTC de pesquisa clínica associada à assistência especializada e de referência da fundação. Em consequência, a partir de 2007 foram criados o Laboratório de Pesquisa Clínica em DST e AIDS (Lapclin-AIDS) e outras subestruturas do INI, igualmente concebidas para assumir vínculos de rápida adaptação - e condicionadas - às demandas e necessidades dos potenciais usuários das suas pesquisas, com vistas a promover a pesquisa inovadora - e a iniciativa empreendedora.

Este estudo sobre a eficácia da estrutura organizacional Adhocrática de laboratórios de pesquisa utiliza, portanto, os procedimentos de apuração de custos do Projeto PAA-IGs e a análise da correlação dos ranks das despesas anuais e dos custos unitários do INI na avaliação do desempenho em custos dessas novas subestruturas da Fiocruz, com o objetivo de avaliar o efeito da adoção da configuração Organização Inovadora no desempenho em custo da pesquisa clínica associada à assistência em saúde, tendo em vista confirmar o crescimento e a efetividade em custos da pesquisa clínica associada à atenção clínica especializada no Lapclin-AIDS (BANA E COSTA, 2000).

Este artigo sobre os resultados do estudo está estruturado em cinco seções, incluindo esta introdução. Primeiro, é apresentada a fundamentação teórica das abordagens utilizadas no estudo acerca das relações entre estrutura organizacional e inovação e das relações entre a escolha do sistema de custeio para a aferição de despesas e custos e a preservação do incentivo à produção dos serviços de atenção integral 
em saúde. O método da pesquisa é descrito na seção três; a análise dos resultados do estudo de caso é feita na seção quatro; e as conclusões, na seção cinco, são seguidas das referências bibliográficas.

\section{Fundamentação Teórica}

Esta seção examina, primeiro, os conceitos de estrutura organizacional e de orientação inovadora presentes na literatura de Administração e Economia interna das organizações. Ao mesmo tempo, ilustra os conceitos pela análise da documentação dos estudos realizados na Fiocruz sobre o tema, que investigaram as relações potenciais entre grau de descentralização e desempenho das subestruturas de laboratórios de pesquisa clínica associada à atenção clínica do INI. Em segundo lugar, introduz os procedimentos de apuração de custos compatíveis com a preservação do incentivo à prestação de serviços de atenção integral em saúde, tal como requer a eficácia do modelo de gestão orientada para resultados, segundo a literatura de Administração e Economia e que, também por este motivo, são incorporados ao Modelo PAA-IGs de apuração de custos.

\section{Estrutura organizacional e inovação}

Mintzberg denominou a forma de alocação e de coordenação da força de trabalho entre as tarefas da organização por "estrutura organizacional". Citou, entre elas, a Burocracia Profissional, a Organização Divisionalizada e a Organização Inovadora (MINTZBERG, 1995).

Por sua vez, a pesquisa sobre a vantagem competitiva da firma produtora focaliza três dimensões da estrutura organizacional que influenciam sua orientação empreendedora: (a) a complexidade - multiplicidade de funções; (b) a formalização das normas; e (c) a centralização - concentração - da tomada de decisão (MORTON; HU, 2008).

De acordo com a literatura, as organizações se confrontam com situações que requerem a atenção do gestor para atividades de orientação empreendedora e que permitam internalizar o espírito empreendedor para habilitar o seu pessoal. Dessa necessidade resulta a importância tanto das organizações que encorajam a iniciativa e o potencial do indivíduo, quanto de uma estrutura organizacional que ofereça condições para motivar a orientação empreendedora (JOHNSON; VAN DE VEN, 2002).

Segundo Lumpkin, Cogliser e Schneider (2010), ainda, a autonomia é a influência direta de maior peso na orientação empreendedora do pessoal, orientação à qual é atribuído importante efeito sobre o desempenho da organização.

Destaca-se, afinal, que a tomada de decisão e a ação individual são influenciadas por características tais como a autonomia e a atitude competitiva, inovadora, pró-ativa e de predisposição ao risco. Combinadas, essas características melhoram o desempenho da organização, que alcança metas com mais eficiência, desafia a concorrência e adquire capacidade adaptativa a ambientes turbulentos (JOGARATNAM; CHINGYICK TSE, 2006).

Uma conclusão importante dessa literatura é que a estrutura organizacional deve ser menos centralizada e envolver mais participação (ROBINSON, 2007). A organização pública que adota a remuneração por desempenho deve levar em conta a estrutura organizacional e descentralizar (CARUANA; EWING; RAMASESHAN, 2002).

Sob esse prisma, a Burocracia Profissional adaptase a um ambiente estável, com sistemas técnicos não regulados e não sofisticados. É uma estrutura organizacional constituída por profissionais com alta padronização das habilidades, em que o trabalho é complexo, prevalecendo a contratação, para as "unidades operacionais", de profissionais treinados e que detêm o controle sobre o seu próprio trabalho, com vistas à internalização dos padrões. $\mathrm{O}$ mecanismo de coordenação típico é a padronização das habilidades (MINTZBERG, 1995).

Uma vez que esses padrões têm origem fora da estrutura, no caso de associações autogovernadas em que os profissionais se reúnem com profissionais de outras associações, a Burocracia Profissional enfatiza a autoridade de natureza profissional e o poder do conhecimento, permitindo elevada autonomia aos profissionais. Nesse tipo de estrutura, há pouca necessidade de supervisão direta sobre os profissionais, ou de ajustamento mútuo entre eles e as unidades operacionais, de hierarquia, de formalização e de planejamento e controle. 
A AC da Fiocruz, por exemplo, uma estrutura típica de Burocracia Profissional, detinha, até 1985, o controle de considerável parte dos recursos e estabelecia as diretrizes, os rumos e o projeto organizacional da Fundação, fixando as prioridades para a alocação de recursos. Apesar disso, caracterizava-se pela baixa governabilidade.

$\mathrm{Na}$ Organização Divisionalizada, em contraposição, a unidade operacional é diferenciada pela padronização dos outputs, o que torna as unidades operacionais suficientemente autônomas: as divisões possuem alta autonomia e baixa hierarquia. A estrutura divisionalizada é adequada à organização constituída por unidades operacionais independentes, agrupadas por um "escritório central". As divisões encontram-se sob controle uniforme do "escritório central" e cada divisão possui sua estrutura própria, diferente das demais (MINTZBERG, 1995). Assim, a intensidade de controle no "escritório central" é relativizada, resultando em descentralização pronunciada: a Organização Divisionalizada exige descentralização e, em princípio, as unidades operacionais adquirem a autonomia necessária para tomar as decisões concernentes às suas próprias operações.

No entanto, como destaca Mintzberg (1995, p. 241), "surge [então] a questão sobre como o escritório central pode manter controle e permitir autonomia suficiente a cada divisão". O "escritório central" deve manter alguma forma de controle sobre as unidades operacionais, sustentando, ao mesmo tempo, a coordenação da organização como um todo. Em geral, o "escritório central" permite ampla autonomia para que as divisões tomem suas próprias decisões e depois monitora os resultados destas decisões.

$\mathrm{Na}$ Organização Divisionalizada, a par de proporcionar autonomia, a padronização dos outputs também funciona como mecanismo de coordenação das unidades operacionais, mas o parâmetro-chave do desenho da organização é o sistema de controle de desempenho.

Face à participação da Fiocruz no desenvolvimento do projeto nacional de C\&T para a área da saúde a partir de 1985, a transição entre as estruturas organizacionais Burocracia Profissional e Organização Divisionalizada foi a forma de fortalecer os dirigentes das UTC através da descentralização administrativa e da informação (FIOCRUZ, 2006).
Em 1985, a competência de ordenar despesas foi delegada aos dirigentes das UTC da Fiocruz. Os processos de compras, de pagamento e outros processos de realização de despesas, no entanto, permaneceram centralizados na AC. Até 1995, os instrumentos de tomada de decisão das UTC ficaram restritos ao papel de organizar as solicitações anuais de recursos públicos.

A partir de 1995, efetivou-se a descentralização administrativa da Fiocruz e a dos processos de compras, orçamentação, contabilidade, tesouraria, almoxarifado e outros para o controle das UTC. O fortalecimento dos dirigentes das UTC resultou na descentralização em estruturas divisionalizadas, com o objetivo de fortalecer a atividade de pesquisa através da promoção da pesquisa estratégica em saúde, a pesquisa orientada para resultados.

Por sua vez, a geração de conhecimento sobre doenças infecciosas foi definida como o produtopadrão da pesquisa estratégica em saúde no INI, a partir da análise do posicionamento estratégico empreendida em 1999. A estratégia adotada no INI para a promoção da pesquisa a partir desse ano (HASSLOCHER-MORENO et al., 2013) consistiu em aproveitar as subestruturas organizacionais de diagnóstico laboratorial e de atendimento préexistentes para implementar Ações Integradas de atividades multidisciplinares de pesquisa clínica, ensino e atenção clínica (PAI).

Em vista dessa transição de estrutura, as UTC passaram a ter autonomia restrita, pois seus outputs são escolhidos a priori, através da definição do plano estratégico acordado com a AC, o chamado Plano Anual (PA), cujo monitoramento pela AC como "escritório central" é formalizado através da avaliação quantitativa do desempenho (JORGE, 2001).

A esse propósito, a atividade de pesquisa, sujeita a incertezas, é realizada em um ambiente dinâmico e, por ser de difícil avaliação, molda um ambiente complexo - marcado pelo conflito de interesses. O ambiente de pesquisa, portanto, também é compatível com o desenvolvimento da estrutura denominada Organização Inovadora (ou Adhocracia Operacional; MINTZBERG, 1995), que exibe estrutura orgânica e descentralização efetiva, focalizada na integração de profissionais de diferentes especialidades em equipes regulares de projetos ad hoc. 
Associada à especialização das tarefas, porém sem padronização das habilidades, e devotada à adaptação rápida aos novos projetos, a Organização Inovadora é uma estrutura que exibe pouca formalização e centralização, planejamento limitado das ações, descentralização seletiva, dispersão da tomada de decisão, delegação do controle aos especialistas e ajuste mútuo como principal mecanismo de coordenação (MINTZBERG, 1995).

Entre as características da estrutura Organização Inovadora que diferem quando comparadas com as das estruturas Burocracia Profissional e Organização Divisionalizada, algumas merecem destaque.

Em primeiro lugar, quanto à interface com o ambiente externo, a formulação de estratégias (a) evolui continuamente, de acordo com os projetos da carteira; (b) emprega analistas do ambiente externo, pesquisadores de marketing e alguma ação de planejamento da própria equipe do projeto; e (c) não depende de habilidades e produtos-padrão ou de sistemas de regulação como mecanismo de coordenação externa.

Em segundo lugar, a tomada de decisão na estrutura Organização Inovadora caracteriza-se por (a) descentralização seletiva; (b) dispersão em todos os níveis; (c) delegação do controle aos especialistas; (d) dedicação de parcela importante do tempo do dirigente ao monitoramento dos projetos, uma vez que o trabalho no projeto inovador é difícil de controlar; e (e) o ajuste mútuo dos profissionais como principal mecanismo de coordenação.

Finalmente, a propósito do mecanismo de conciliação entre os objetivos do núcleo operacional e da administração, cuja importância nas organizações de saúde é destacada por Harris (1977), a resposta à mudança externa pelo núcleo operacional reduz a necessidade de supervisão direta dos operadores pelos gerentes de linha na Organização Inovadora, que são incorporados às equipes de projetos como membros funcionais.

Efetivada a partir de 2007, a reestruturação orientada para a promoção da inovação das UTC da Fiocruz através de uma nova combinação de descentralização com redução da complexidade hierárquica e da formalização das normas da tomada de decisão sobre a atividade de pesquisa perseguiu maior interação dos profissionais, por ajuste mútuo, em equipes de projetos estruturadas. A organização posterior dos laboratórios da Fundação resultou da busca de adequação das suas características às da Adhocracia Operacional.

O diagnóstico subjacente à mudança, completado em 2006, assinalou, em primeiro lugar, o avanço na padronização dos processos gerenciais e produtos da Fiocruz e também da diversificação da participação das UTC na produção (FIOCRUZ, 2006). Apontou, no entanto, a ausência de configurações matriciais para a produção por meio de redes internas e de estruturas horizontais de coordenação para substituir as estruturas verticais adotadas em cada UTC, independentemente da predominância de atividades de pesquisa, ensino, produção ou atenção clínica, apesar de certa "coexistência dos diferentes modelos formulados por Mintzberg” (FIOCRUZ, 2006, p. 11).

Em terceiro lugar, quanto à pesquisa clínica associada à atenção clínica especializada e de referência nas UTC tais como o INI, identificou uma configuração mais próxima do modelo burocrático profissional para as atividades predominantemente assistenciais e "bem mais próxima do modelo Adhocrático típico para atividades onde existe uma maior necessidade de ajustamento mútuo entre a assistência e a pesquisa clínica" FIOCRUZ (2006, p. 14).

Nesse sentido, a integração das atividades de atenção clínica, ensino e pesquisa do INI através das PAI, preconizada pelo Modelo de Atenção Integral em saúde, dependia da promoção, por ajuste mútuo, da interação efetiva entre profissionais com diferentes padrões de habilidades (HARRIS, 1977).

Em consequência, a diretriz originária da estratégia de mudança para as UTC como o INI foi a

estruturação das ações assistenciais entre as modalidades (internação, hospital-dia e ambulatório), as linhas de cuidado (ginecologia, obstetrícia, neonatologia, pediatria, doenças infecciosas) e o âmbito das atividades de apoio (nutrição e alimentação; patologia clínica; assistência social; etc.) e de suporte teórico (epidemiologia clínica, bioestatísticas e informática), perpassando e integradas em projetos de pesquisa clínica e ensino, sempre que procedente, FIOCRUZ (2006, p. 14). 
Por sua vez, quanto aos instrumentos de implementação dessa estratégia: (a) "os elementos de ligação (das instâncias matriciais) passaram a ter maior relevância estratégica que as estruturas hierárquicas em si mesmas" FIOCRUZ (2006, p. 12); (b) à “tecnoestrutura (...), relativamente reduzida" FIOCRUZ (2006, p. 12), coube incorporar "as ações de planejamento estratégico, com forte foco em objetivos e projetos horizontais" FIOCRUZ (2006, p. 12); (c) “a estruturação de uma Assessoria de Apoio forte (visou) liberar os profissionais do núcleo operacional (pesquisadores e professores) de executar atividades gerenciais rotineiras" FIOCRUZ (2006, p. 11); e (d) o laboratório de pesquisa seria a subestrutura geradora de conhecimento, que pode resultar em métodos, processos, tecnologias e outros produtos, "(b)asicamente (...) formado por um ou mais grupos de pesquisa, segundo reconhecimento do Diretóriodos Grupos de Pesquisa do CNPq" FIOCRUZ (2006, p. 12), uma equipe técnico-científica, portanto. Além disso, deveria constituir "o núcleo principal da organização da pesquisa e o desenvolvimento tecnológico, estando a ele freqüentemente associadas responsabilidades de serviços de referência laboratorial e do ensino ou organização de coleções" FIOCRUZ (2006, p. 12).

Finalmente, os mecanismos de incentivo à orientação inovadora das UTC consistiriam, primeiro, de novas regras de constituição ad hoc dos laboratórios de pesquisa e, segundo, de novas regras sobre a sua existência transitória.

Assim, a subestrutura Laboratório de Pesquisa foi concebida para assumir, na Fiocruz,

vínculos adhocráticos de ajustamento mútuo entre si e com relação aos espaços de ligação programática da tecnoestrutura e de assessoria de apoio gerencial e tecnológico que lhe permitem rápida adaptação às demandas e necessidades dos potenciais usuários das suas pesquisas, FIOCRUZ (2006, p. 22).

Os critérios e mecanismos regulatórios internos para a habilitação e reconhecimento de um laboratório de pesquisa na Fiocruz incluiriam, a propósito: "(a) valiação ex-ante periódica, que considere a vinculação dos projetos propostos com prioridades institucionais (...) e com metas estratégicas (...)"FIOCRUZ (2006, p. 13); (a)valiação ex-post periódica, com base em indicadores de eficácia, eficiência e efetividade para os projetos propostos; e "(n)úmero limite máximo de laboratórios por (UTC), a ser negociado" FIOCRUZ
(2006, p. 13) entre a AC e as UTC.

Face, portanto, aos objetivos da reestruturação da atividade de pesquisa do INI, a avaliação da eficácia da estrutura de laboratório de pesquisa clínica adotada está relacionada, neste artigo, à obtenção de indicadores sobre o desempenho das atividades e sobre as despesas de produção que permitam confirmar o crescimento e a efetividade em custos da pesquisa clínica associada à atenção clínica especializada e de referência.

\section{Aferição das estimativas de despesas e custos e incentivo}

De fato, a consistência entre a doutrina da administração pública gerencial (BRESSER PEREIRA, 1996) e a adoção do modelo de gestão orientada para resultados nas organizações públicas (ROVERE, 1997) depende da preservação da propriedade de incentivo do sistema de apropriação de custos aos produtos e serviços produzidos utilizado na organização (BIRKINSHAW; HAMEL; MOL, 2008).

Como corolário dos princípios da administração pública gerencial, o custo apropriado em cada tipo de bem ou serviço produzido - o seu custeio - deve, em particular, assegurar o incentivo suficiente para que a tomada de decisão na organização pública resulte na escolha correta dos produtos e a quantidade que vai ser produzida, a partir da remuneração adequada e da satisfação das preferências do comprador (ELDENBURG; WOLCOT'T, 2007).

No entanto, a primeira dificuldade para a escolha do sistema de apropriação de custos da organização pública multipropósito decorre da sua frequente participação em mercados em que o desconhecimento dos preços competitivos dificulta a tomada de decisão sobre quais produtos, quais quantidades e a que custo produzir (BISANG; KATZ, 1996).

Um caso típico do segundo problema enfrentado quando da escolha do incentivo correto ao produtor através da remuneração do seu produto - ou da parcela do custo do seu produto reconhecida no ato de compra - é o da prestação de serviços de saúde (ALMEIDA; BORBA; FLORES, 2009). A satisfação proporcionada por essa atividade depende da experiência de uso, o que turva a sinalização "a priori" da qualidade do serviço, necessária à eficiência das trocas. 
$\mathrm{Na}$ administração pública, o sistema de Custeio Baseado em Atividades (Activity-Based Costing - ABC) é um método de custeio orientado para diminuir as distorções provocadas pelo rateio arbitrário dos custos indiretos. Para Nakagawa (1994), esse método foi desenvolvido para facilitar a análise estratégica dos custos relacionados com as atividades que mais impactam o consumo de recursos de uma empresa. Atende uma necessidade informacional das organizações com alta participação de custos indiretos e com variado mix de produtos ou serviços (SOUZA; MAUSS; DIEHL, 2008). Em organizações públicas de saúde existem vários tipos de atividades, o que justifica usar o $\mathrm{ABC}$.

Para Martins (2008, p.297), os conceitos e a metodologia do Custeio Baseado em Atividades aplicam-se também às empresas não industriais, sendo possível utilizálo em instituições financeiras, concessionárias de serviços públicos, hospitais, escolas, enfim, em todos os tipos de organizações, inclusive naquelas sem fins lucrativos.

Segundo Heitger, Logan e Matulich (1992 apud SOUZA et al., 2009, p. 18), não é possível realizar uma avaliação de desempenho adequada sem dispor de informações sobre custos. Em particular, o sistema ABC destaca-se como uma das ferramentas comumente utilizadas para a gestão de custos. Esse sistema visa alocar os custos dos recursos às atividades e, destas, aos produtos/serviços, de modo a diminuir/ eliminar as informações distorcidas apresentadas pelos sistemas de custeio tradicionais (MOHAN; PATIL, 2003 apud SOUZA et al., 2009, p. 18).

O aperfeiçoamento do levantamento de despesas em nível de microcustos (MEDICI; MARQUES, 1996) e da apuração de custos pelo método $\mathrm{ABC}$ é, portanto, um mecanismo pró-eficiência da gestão das organizações públicas multipropósito de saúde (CUNILL GRAU; OSPINA BOZZI, 2003). Segundo Bonacin e Araújo (2011, p.1065), ademais, os sistemas gerenciais devem ser tempestivos e dinâmicos, como são as instituições e também fomentar a melhoria continua.

O modelo de avaliação do desempenho em custos do INI proposto pelo Projeto PAA-IGs considera, em primeiro lugar, que a gestão estratégica dos seus custos está focalizada na PAI - agora designada laboratório de pesquisa clínica associada à assistência especializada e de referência, a subestrutura que: integra as suas atividades de diagnóstico laboratorial, atendimento clínico, ensino e pesquisa de doenças infecciosas; promove a pesquisa estratégica sobre saúde; acumula reputação para a organização; reforça o seu posicionamento estratégico; e, desta maneira, mobiliza recursos adicionais para a pesquisa clínica de doenças infecciosas do INI (JORGE et al., 2006).

Face às exigências de capacidade contínua de adaptação e mudança descritas, em suma, a gestão estratégica de custos (WARREN; REEVE; FESS, 2008) no INI através dos métodos de levantamento de microcustos (HORNGREN; SUNDEM; STRATTON, 2004) e de custeio ABC hoje conta com o suporte de planilhas flexíveis de armazenamento e processamento dos dados básicos do Projeto PAA-IGs em linguagem Excel, porque: (a) a estrutura organizacional do INI é dinâmica; (b) a criação de novas subestruturas do INI reorganizam as atividades de diagnóstico, atenção clínica, ensino e pesquisa de diferentes maneiras; (c) a criação de novas subestruturas altera as regras de apropriação dos custos comuns (apoio administrativo interno, administração do Campus e atividades de suporte das atividades) entre os distintos serviços de atenção integral em saúde do INI (por exemplo, atenção clínica, egressos de cursos e produção bibliográfica); (d) o conhecimento do custo efetivo médio de cada tipo de serviço prestado das subestruturas do INI depende de novos cálculos toda vez que algum laboratório de pesquisa clínica, especialidade, tipo de exame, serviço de atenção clínica e insumo é incluído ou excluído da combinação de recursos e da pauta de produção do INI; e (e) os indicadores de desempenho em custos das subestruturas do INI devem ser atualizados periodicamente (BRIERS; HIRST, 1992).

\section{Método de Pesquisa}

Tendo em vista o objetivo de investigar o efeito da reestruturação dos laboratórios do INI segundo o modelo Organização Inovadora de Mintzberg (1995) sobre a produção da pesquisa e o desempenho em custos da atenção clínica nestas subestruturas organizacionais, o método escolhido para esta investigação é o desenvolvimento de um estudo de caso único sobre a evolução do Lapclin-AIDS a partir da sua reestruturação entre 2006 e 2009. Esse método foi escolhido para esta pesquisa empírica quali-quantitativa de tipo descritivo, porque analisa, sob a ótica do observador externo, os dados 
recentes de uma experiência pioneira, para agregar conhecimento sobre o efeito da descentralização. Vale dizer, o conhecimento existente a respeito desse efeito é insuficiente para confirmar relações de causalidade entre o incentivo à orientação inovadora $\mathrm{e}$ o desempenho da pesquisa clínica associada à atenção clínica (YIN, 2005).

Inicialmente o estudo identifica os procedimentos necessários à incorporação do Lapclin-AIDS, o maior laboratório de pesquisa clínica do INI, na representação dos atuais 16 laboratórios existentes no Instituto, com vistas ao suporte empírico da análise de desempenho em custos proposta no Projeto PAAIGs, a partir do levantamento das despesas em nível de microcustos e da apuração dos custos unitários efetivos, com base nos princípios do Método ABC.

De forma a conhecer o tipo de associação de fato existente entre Despesa Direta anual e custo unitário efetivo por serviço de atenção clínica de referência de diagnóstico e de atendimento no INI, a análise do desempenho em custos dos laboratórios é feita, por sua vez, em duas etapas. Na primeira se examina, com auxílio do Coeficiente de Correlação de Spearman (SIEGEL, 1956), a correlação estatística dos ranks dos valores das despesas anuais e dos custos unitários, por tipo de serviço de atenção clínica prestado pelo LAPCLIN-AIDS em 2009, e das suas variações entre 2006 e 2009, ou seja, antes e depois da reestruturação. $\mathrm{Na}$ segunda se analisa o desempenho em custos da nova estrutura organizacional do INI por meio do exame da correlação estatística dos ranks dos diferentes valores das despesas e dos custos dos nove laboratórios de pesquisa clínica mais estruturados do INI em 2009.

Com vistas a cumprir objetivos e metas das atividadesfim de ensino, pesquisa e atenção clínica, cada laboratório de pesquisa clínica do INI, doravante a subestrutura representativa da PAI correspondente neste estudo, focaliza uma nosologia específica e engloba atividades especializadas de: diagnóstico de laboratório; atendimento de ambulatório, hospitaldia e de internação; suporte exclusivo, seja do ensino, seja da pesquisa; e apoio administrativo interno indistinto ao conjunto das atividades especializadas. Aborda, além disso, os diferentes aspectos relativos às múltiplas especialidades profissionais mobilizadas segundo a abordagem do Modelo de Atenção Integral em saúde (HASSLOCHER-MORENO et al., 2013).
Ao considerarmos a coorte de pacientes composta e acompanhada em um banco de dados dos projetos de pesquisa dos médicos infectologistas do INI como referência para demarcar o que é entendido como um laboratório de pesquisa clínica neste estudo, cada laboratório inclui todas as atividades de pesquisa, de ensino, de diagnóstico e de atenção clínica do INI. Vistos em conjunto, esses laboratórios, vale dizer, respondem quase que integralmente pelo conjunto das atividades do INI de per si. A participação do Lapclin-AIDS é expressiva, representando 49,9\% dos pacientes em coorte, $54,6 \%$ das consultas agendadas, $73 \%$ dos atendimentos de hospital-dia, 58,3\% dos dia/ ano de internação e 46,9\% dos exames de diagnóstico.

O Lapclin-AIDS compartilha uma peculiaridade com outros laboratórios de pesquisa clínica do INI, pois realiza serviços de referência de natureza distinta, que englobam, em simultâneo, seis tipos de consultas e um tipo de procedimento médico - entre consultas de médicos infectologistas, médicos de outras especialidades e clínicos complementares, dois tipos de coleta de material - Coleta de Sangue e de Urina, dois tipos de exames de diagnóstico realizados internamente - Teste Rápido para Detecção do HIV e Teste de Gravidez, e seis tipos de procedimentos Armazenamento de Alíquotas de Soro, Plasma, Urina, Sêmen, Células e Secreção Vaginal.

Face a essa diversidade dos serviços de referência prestados no Lapclin-AIDS, vários itens de despesa inscritos no orçamento do INI e que compõem a Despesa Direta de cada serviço de referência concorrem, em primeiro lugar, para a formação do custo unitário efetivo dos serviços componentes destas atividades, a partir das despesas em nível de microcustos: a despesa de Kits \& Reagentes; a despesa de Serviços de Terceiros - Pessoa Física; a despesa de material de almoxarifado; a despesa de material de consumo não-almoxarifado; a despesa de telefonia; a despesa de amortização de material permanente; a despesa de manutenção de material permanente e a despesa direta de apoio administrativo interno.

Em decorrência da incorporação do Lapclin-AIDS à representação da estrutura organizacional do INI pelo Projeto PAA-IGs, a adaptação das planilhas estruturadas de cálculo dos custos unitários efetivos dos serviços prestados nas subestruturas do Instituto pelo projeto envolveu as seguintes etapas:

(a) mapeamento dos insumos e serviços do Lapclin- 
AIDS e incorporação à representação do INI objeto do método do Projeto PAA-IGs;

(b) levantamento das despesas do Lapclin-AIDS em nível de microcustos (BREWER; NORREN; GARRISON, 2005);

(c) levantamento dos preços de aquisição e das quantidades anuais gastas dos insumos do LapclinAIDS;

(d) incorporação do Lapclin-AIDS às planilhas de apuração de custos pelo método do Projeto PAA-IGs;

(e) cálculo das novas proporções de participação dos serviços prestados no INI na apropriação dos custos comuns;

(f) cálculo da Despesa Direta de cada tipo de serviço prestado no INI; e

(g) cálculo das despesas anuais e unitárias médias dos laboratórios de pesquisa clínica, das atividades e dos serviços prestados do INI a preços de 2009.

Quando do levantamento dos valores das despesas e das quantidades anuais da produção física de serviços do Lapclin-AIDS, os custos unitários das atividades foram apurados como custos médios, já que as atividades compartilham custos comuns, são sujeitas a contingências do quadro nosológico e não obedecem rotinas-padrão. Os dados de produção, despesa e material permanente, expurgados de gastos sazonais, referem-se ao período anual.

Foram utilizados dados secundários coletados nos arquivos centrais do sistema de informação do INI Sistema de Controle do Centro de Clínicas (CECLIN) e Sistema de Controle do Centro Laboratorial (SERVLAB) - ou obtidos mediante consulta direta aos arquivos do Serviço de Informação Hospitalar (SIH) e às demais subestruturas do INI, com o auxílio dos instrumentos de coleta do Projeto PAA-IGs.

\section{Análise dos Resultados}

Esta seção analisa a evolução do volume de atividades e do desempenho em custos do Lapclin-AIDS a partir da reestruturação do laboratório em 2007.

Como a Tabela 1 permite visualizar, houve, em primeiro lugar, um crescimento do número de projetos de pesquisa clínica em desenvolvimento no LapclinAIDS de cerca de $80 \%$, do número de consultas de cerca de $46 \%$ e dos dias de internação de cerca de $50 \%$ no acumulado do período 2006-2009, indicativo do efeito positivo sobre a produção do laboratório logo no primeiro triênio subsequente à sua reestruturação (HASSLOCHER-MORENO et al., 2013).

Tabela 1: LAPCLIN-AIDS - Quantidade Anual por Atividade - 2006-2009

\begin{tabular}{lll}
\hline \multirow{2}{*}{ Atividade } & \multicolumn{2}{c}{ Quantidade-ano } \\
& $\mathbf{2 0 0 6}$ & $\mathbf{2 0 0 9}$ \\
\hline $\begin{array}{l}\text { Ambulatório } \\
\text { Consulta de Infectologista }\end{array}$ & 6.357 & 10.179 \\
$\begin{array}{l}\text { Consulta de Médico Não- } \\
\text { Infectologista }\end{array}$ & 1.070 & 1.872 \\
Consulta de Outras & & \\
Especialidades & 6.481 & 8.222 \\
$\quad$ Procedimentos de & & \\
Enfermagem & 4.208 & 3.641 \\
Internação & 2.766 & 4.142 \\
Hospital-dia & 2.236 & 1.671 \\
Ensino (1) & 18 & 19 \\
Pesquisa (2) & 32 & 58 \\
\hline
\end{tabular}

Fonte: Dados da Pesquisa.

(1) Número de egressos de cursos.

(2) Número de projetos de pesquisa em desenvolvimento.

Uma vez identificados, à luz dos princípios destacados em Birkinshaw, Hamel e Mol (2008) e Nakagawa (1994), os itens de despesa componentes do custo dos serviços definidos na seção precedente e levantada a quantidade de serviços prestada pelo laboratório em 2009, o resultado da aplicação dos princípios do Projeto PAAIGs à apuração das despesas anuais e dos custos unitários efetivos com recursos do orçamento do INI (Despesa Direta) por serviço de referência é apresentado na Tabela 2. O custo unitário efetivo médio e a quantidade anual produzida de cada serviço em 2009 são usados para calcular as despesas anuais por tipo de serviço constantes das colunas ao final da tabela.

Concluído o cálculo do custo unitário efetivo e da despesa anual de cada serviço de referência prestado no Lapclin-AIDS, uma primeira questão de interesse sobre o efeito da reestruturação organizacional do laboratório no desempenho em custos das suas atividades de atenção clínica é a que diz respeito ao possível efeito adverso da diversificação dos serviços - a busca de integralidade (BISANG; KATZ, 1996) sobre a efetividade em custo destas atividades. 
Tabela 2: LAPCLIN-AIDS - Custo Unitário Efetivo e Despesa Anual com Recursos do Orçamento do INI por Serviço de Referência - 2009 (1)

\begin{tabular}{|c|c|c|c|}
\hline \multirow[b]{2}{*}{ Serviço de Referência } & \multirow[b]{2}{*}{ Quantidade } & \multicolumn{2}{|c|}{ Despesa Direta } \\
\hline & & $\begin{array}{c}\text { Custo Unitário } \\
\text { Efetivo }\end{array}$ & Despesa Anual \\
\hline \multicolumn{4}{|l|}{ CONSULTAS } \\
\hline \multicolumn{4}{|l|}{ Infectologista } \\
\hline Consulta Ambulatorial de tipo Agendada & 9.038 & 55,86 & $504.898,82$ \\
\hline Consulta Ambulatorial de tipo PAT & 1.141 & 54,09 & $61.695,31$ \\
\hline Procedimento Ambulatorial & 3 & 67,99 & 203,96 \\
\hline \multicolumn{4}{|l|}{ Médico não-Infectologista } \\
\hline Consulta Ambulatorial de tipo Agendada & 1.821 & 48,05 & $87.509,19$ \\
\hline Consulta Ambulatorial de tipo PAT & 51 & 59,11 & $2.990,89$ \\
\hline \multicolumn{4}{|l|}{ Clínico Complementar } \\
\hline Consulta Ambulatorial de tipo Agendada & 7.418 & 65,25 & $484.061,34$ \\
\hline Consulta Ambulatorial de tipo PAT & 804 & 55,21 & $44.390,38$ \\
\hline \multicolumn{4}{|l|}{ COLETAS } \\
\hline Coleta de Sangue & 4.671 & 41,56 & $194.139,68$ \\
\hline Coleta de Urina & 474 & 40,74 & $19.309,15$ \\
\hline \multicolumn{4}{|l|}{ EXAMES } \\
\hline Teste rápido HIV & 1.675 & 41,83 & $70.057,90$ \\
\hline Teste de Gravidez & 513 & 40,59 & $20.824,42$ \\
\hline \multicolumn{4}{|l|}{ PROCEDIMENTOS } \\
\hline Armazenamento de Alíquotas Soro & 7.876 & 42,84 & $337.373,51$ \\
\hline Armazenamento de Alíquotas Plasma & 17.109 & 42,84 & $732.874,99$ \\
\hline Armazenamento de Alíquotas Urina & 552 & 42,84 & $23.645,27$ \\
\hline Armazenamento de Alíquotas Sêmen & 173 & 42,84 & $7.410,57$ \\
\hline Armazenamento de Alíquotas Células & 3.591 & 51,81 & $186.060,19$ \\
\hline Armazenamento de Alíquotas Secreção Vaginal & 144 & 282,68 & $40.706,58$ \\
\hline
\end{tabular}

Fonte: Dados da Pesquisa.

(1) $\mathrm{Em} \mathrm{R} \$$ a preços de 2012.

Com vistas a examinar o efeito da diversificação do Lapclin-AIDS, a Tabela 2 constitui um ponto de partida. Em princípio, essa segunda tabela permite investigar a relação existente entre as diferenças de custo unitário efetivo desses serviços de referência de diagnóstico e de atenção clínica em nível de ambulatório (conceito Despesa Direta) no ano 2009 e as diferenças no valor da despesa direta anual despendida por serviço de referência.

Dessa forma, a Tabela 2 serve ao propósito exploratório de verificar se ao maior custo unitário por tipo desses serviços de referência correspondeu, em 2009, menor dotação de recursos do orçamento do INI, quando em comparação com as dotações destinadas aos demais tipos de serviços de referência. Isso porque, nesse caso, os ranks das despesas diretas anuais e dos custos unitários dos serviços prestados correspondem a um padrão de distribuição dos recursos do orçamento do INI que é efetivo em custo. Se, ao contrário, esses ranks guardam entre si uma relação muito diferente dessa relação inversa de variação, indicam que a distribuição dos recursos do orçamento do INI por serviço de referência em 2009 não revelou escolha efetiva em custos (JORGE et al., 2006).

Não obstante, é difícil interpretar a Tabela 2 através desse procedimento de observação direta, de forma a 
conhecer o tipo de associação de fato existente entre despesa direta anual e custo unitário efetivo por serviço de referência de diagnóstico e de atenção clínica de nível de ambulatório do Lapclin-AIDS. Uma alternativa é examinar a correlação estatística dos ranks desses valores de despesas e custos para 2009 com o auxílio do Coeficiente de Correlação de Spearman (SIEGEL, 1956).

Em segundo lugar, portanto, a análise dos dados indica que, medida pelo Coeficiente de Correlação de Spearman, a associação da despesa direta anual com o custo unitário efetivo por serviço de referência de diagnóstico e de atenção clínica de nível de ambulatório prestado no Lapclin-AIDS no ano de 2009 é positiva $(\mathrm{R}=+0,037)$, em que pese a baixa precisão $(p>5 \%)$ do valor obtido (ZAR, 1972). Em outros termos, a distribuição dos recursos do orçamento do Instituto entre esses 17 tipos de serviços de atenção à coorte de pacientes do LapclinAIDS revelou-se relativamente pouco efetiva em custo (correlação positiva baixa) em 2009, quando a avaliação desconsidera, em simultâneo, as atividades de pesquisa e ensino do laboratório e o conjunto das atividades dos outros 15 laboratórios de pesquisa do INI (JORGE et al., 2006).

Em terceiro lugar, o cálculo das despesas segundo o critério de apropriação dos recursos inscritos no orçamento do INI (DD) conclui, na Tabela 3, a apresentação dos resultados da análise sob a ótica da minimização de custos, com um primeiro diagnóstico agregado sobre a evolução do desempenho em custos do conjunto dos serviços em cada atividade de atenção clínica do Lapclin-AIDS entre 2006 e 2009: os custos unitários efetivos das consultas de infectologistas, dos procedimentos de enfermagem e do dia de internação caíram, mas não os custos unitários efetivos dos outros procedimentos investigados.

Tabela 3: LAPCLIN-AIDS - Despesa Anual e Custo Unitário por Atividade - 2006-2009

\begin{tabular}{|c|c|c|c|c|c|}
\hline \multirow{2}{*}{ Atividade } & \multicolumn{2}{|c|}{ DD-ano (1), (2) } & \multicolumn{3}{|c|}{ DD/Unidade-serviço (1) } \\
\hline & 2006 & 2009 & 2006 & 2009 & $2009 / 2006$ \\
\hline AMBULATÓRIO & $1.008 .391,74$ & $1.470 .920,56$ & .. & .. & n.a. \\
\hline Consulta de Infectologista & $499.443,16$ & $667.449,83$ & 256,33 & 64,76 & DIMINUIU \\
\hline Consulta de Médico Não-Infectologista & $73.414,25$ & $106.609,41$ & 60,29 & 63,12 & AUMENTOU \\
\hline Consulta de Outras Especialidades & $262.321,25$ & $622.517,93$ & 58,97 & 70,96 & AUMENTOU \\
\hline Procedimentos de Ambulatório & 23,61 & 32,71 & 0,11 & 1,79 & AUMENTOU \\
\hline Procedimentos de Enfermagem & $173.189,47$ & $74.310,69$ & 41,16 & 20,41 & DIMINUIU \\
\hline INTERNAÇÃO & $2.292 .442,40$ & $2.859 .247,36$ & 828,79 & 690,31 & DIMINUIU \\
\hline HOSPITAL-DIA & $268.932,10$ & $383.996,70$ & 120,27 & 229,80 & AUMENTOU \\
\hline Somatório & 4.578.157,98 & $6.185 .085,18$ & $1.365,92$ & $1.141,14$ & DIMINUIU \\
\hline
\end{tabular}

Fonte: Dados da Pesquisa.

Notas:

.. Não se aplica dado numérico.

(1) $\mathrm{Em} \mathrm{R} \$$ a preços de 2012.

(2) DD - Despesa Direta (inscrita no orçamento do INI; sob o controle do gerente do INI).

Finalmente, a comparação da variação da despesa direta anual entre os laboratórios de pesquisa clínica do INI em cada tipo de atividade de atenção clínica com a variação do custo unitário efetivo correspondente no ano de 2009 foi possível a partir dos dados da Tabela 4. Aqui também, com o propósito exploratório de verificar se ao maior custo unitário incorrido pelo laboratório de pesquisa correspondeu menor dotação de recursos do orçamento do INI em 2009, quando comparada com as dotações dos demais laboratórios para a atividade. 
A partir dos dados da Tabela 4, foi então testada a hipótese de que, em 2009, a despesa em cada atividade de atenção clínica do Lapclin-AIDS - atividades de ambulatório (AMB), hospital-dia (HD), internação (INT) e tratamento intensivo (CTI) - foi maior nas atividades com custo unitário menor vis-à-vis o custo unitário dos demais laboratórios.

Tabela 4: INI - Distribuição da Despesa e Custo Unitário por Laboratório de Pesquisa Clínica - 2009

\begin{tabular}{lrrrrrrrr}
\hline \multirow{2}{*}{ Laboratório } & \multicolumn{9}{c}{ Custo Unitário Efetivo (1) } & \multicolumn{1}{c}{ Despesa Direta (1) } & \multicolumn{1}{c}{ CTI } \\
& AGD & \multicolumn{1}{c}{ AHD } & \multicolumn{1}{c}{ TINT } & \multicolumn{1}{c}{ CTI } & \multicolumn{1}{c}{ AMB } & \multicolumn{1}{c}{ HD } & \multicolumn{1}{c}{ INT } & \multicolumn{1}{c}{ (122.275,31 } \\
\hline CHAGAS & 19,42 & 257,68 & 685,10 & 933,40 & $41.981,34$ & $19.325,63$ & $320.627,05$ & - \\
DFA/DENGUE & 102,56 & - & 671,95 & 881,09 & $42.483,63$ & $69.211,08$ & $29.957,05$ \\
OUTRAS DIPs & 145,73 & 197,36 & 672,98 & 885,33 & $81.677,62$ & $10.657,24$ & $470.411,65$ & $162.016,17$ \\
HIV & 65,81 & 229,80 & 690,31 & 962,74 & $594.772,54$ & $383.996,70$ & $2.859 .247,36$ & $981.033,51$ \\
HTLV & 106,34 & 230,31 & 689,51 & 919,67 & $42.257,74$ & $25.564,62$ & $195.820,82$ & $128.754,35$ \\
LTA & 136,45 & 206,60 & 681,49 & - & $56.387,58$ & $22.726,50$ & $34.756,19$ & - \\
MICOSES & 114,44 & 197,31 & 676,59 & 898,91 & $191.714,45$ & $38.081,30$ & $279.433,28$ & $79.104,34$ \\
TOXO & 78,80 & 287,57 & - & - & $40.827,44$ & $3.738,35$ & - & - \\
TB & 70,41 & 229,87 & 675,34 & 891,76 & $129.872,89$ & $14.251,79$ & $550.399,83$ & $249.693,69$ \\
\hline
\end{tabular}

Fonte: Dados da Pesquisa.

Nota:

- Dado numérico igual a zero não resultante de arredondamento.

(1) Em R\$ a preços de 2012.

Examinada a correlação estatística dos ranks desses tipos de despesas com auxílio do Coeficiente de Correlação de Spearman, o quarto resultado da análise dos dados, destacado na Tabela 5, é que a associação da despesa anual com a despesa média das atividades revelou-se negativa no atendimento de ambulatório, internação, CTI e hospital-dia em 2009. Vale notar que, face ao pequeno número de laboratórios já estruturados, de interesse da análise $(n=9)$, apenas o valor de um dos coeficientes obtido é significativo a 5\% ou menos (ZAR, 1972).

Tabela 5: INI - Efetividade em Custo da Distribuição da Despesa da Atividade por Laboratório de Pesquisa Clínica - 2009

\begin{tabular}{|c|c|c|c|c|c|c|}
\hline \multirow{2}{*}{\multicolumn{2}{|c|}{ R, RE (1) }} & \multicolumn{5}{|c|}{ DD-ano: 2009} \\
\hline & & Ambulatório & Hospital-Dia & Internação & CTI & \\
\hline \multirow{4}{*}{ 商芦 } & Ambulatório & $-0,13(\mathrm{R})$ & .. & .. & & .. \\
\hline & Hospital-Dia & .. & $-0,02(\mathrm{R})$ & .. & & .. \\
\hline & Internação & .. & .. & $-0,45(\mathrm{R})$ & & .. \\
\hline & CTI & .. & .. & .. & & $-0,73(\mathrm{RE})^{* *}$ \\
\hline
\end{tabular}

Fonte: Elaboração dos autores.

Nota:

.. Não se aplica dado numérico.

$* *: \mathrm{p}<5 \%$

(1) R, RE - Coeficiente de Correlação de Spearman, respectivamente sem e com empate de postos. 


\section{Conclusões}

Como geração de conhecimento, este estudo com foco na organização pública de pesquisa clínica associada à assistência, contribuiu para a formulação de hipóteses sobre o desempenho em custos das organizações públicas multipropósito de saúde, assim como para a adaptação do marco analítico aplicável à avaliação da eficácia de estruturas organizacionais à discussão da inovação e para a sistematização de princípios e procedimentos aplicáveis à apuração de despesas e custos neste tipo de organização.

O resultado da análise de eficácia do modelo de reestruturação do INI (BANA E COSTA, 2000) permitiu concluir que, face aos objetivos do modelo Adhocrático de estrutura organizacional divisado por Mintzberg (1995), o crescimento da atividade de pesquisa do Lapclin-AIDS, após adotada a nova configuração, não assegurou efetividade em custos pois, avaliados individualmente, alguns procedimentos de atenção clínica revelaram-se efetivos em custo em 2009 e outros não.

No sentido da análise microeconômica, também para o período da transição para a nova estrutura organizacional do INI, o período 2006-2009, não foi possível confirmar a hipótese de comportamento poupador de custos, tal como inspirada na teoria da produção em concorrência perfeita. A preços de 2012, a expansão do Lapclin-AIDS ocorreu com redução dos custos unitários de vários procedimentos de atenção ambulatorial do laboratório, mas não da consulta prestada por médicos não-infectologistas, da consulta de especialidades não-médicas, dos procedimentos de ambulatório e do atendimento de hospital-dia.

Não obstante, sob a ótica do desempenho em custos do conjunto dos laboratórios, vale dizer, da estrutura organizacional do INI, o resultado da análise não permite confirmar a hipótese nula de inexistência de associação entre a reestruturação dos laboratórios e a efetividade em custos da distribuição dos recursos orçamentários de cada atividade de atenção clínica do INI entre os laboratórios. O resultado do cálculo dos indicadores de custo unitário efetivo da atividade de atenção clínica foi de que (a) a promoção do crescimento da pesquisa do INI ocorreu sem prejuízo da efetividade em custo das suas atividades de atenção clínica e (b) a distribuição dos recursos destinados às atividades de atenção clínica fortaleceu os laboratórios mais eficientes.

Com respeito à adequabilidade do modelo do Projeto PAA-IGs do INI frente à perspectiva de mudança Adhocrática da estrutura organizacional da pesquisa clínica associada à assistência especializada e de referência em saúde, ao incorporar procedimentos de levantamento das despesas em nível de microcustos e princípios de apropriação de custos compatíveis com o método $\mathrm{ABC}$ ao projeto, o estudo contribuiu fornecendo conhecimento sobre inventário de dados básicos, plano de contas e planilhas estruturadas utilizados.

Como contribuição gerencial, o estudo confirmou a compatibilidade da nova estrutura com a efetividade em custo da atenção clínica, e a flexibilidade do método do Projeto PAA-IGs para a aferição do custeio dos serviços do INI face à reestruturação, complementando o plano tabular, os canais de levantamento de dados básicos, e as planilhas de apuração de orçamentos do projeto (JORGE et al., 2010).

Como geralmente ocorre em estudos de caso, a análise de um único Instituto de Pesquisa e de um pequeno número de laboratórios de pesquisa impõe uma limitação à generalizabilidade dos resultados obtidos quanto ao efeito da mudança de estrutura organizacional sobre o dinamismo da inovação e o desempenho em custos dos seus laboratórios. Essa restrição, no entanto, somente poderá ser contornada mediante esforços futuros de pesquisa sobre uma amostra maior de laboratórios para validações adicionais do modelo do Projeto PAA-IGs, estimulando a sua difusão entre outras organizações de saúde.

\section{Referências}

ALMEIDA, A. G.; BORBA, J. A.; FLORES, L. C. S. A utilização das informações de custos na gestão da saúde pública: um estudo preliminar em secretarias municipais de saúde do estado de Santa Catarina. Revista de Administração Pública, v. 43, n. 3, p. 579-607, mai./jun. 2009.

BANA E COSTA, C. Entrevista, Boletim da SOBRAPO, Rio de Janeiro, n. 20, fev. 2000. 
BIRKINSHAW, J.; HAMEL, G.; MOL, M. J. Management innovation. Academy of Management Review, v. 33, n. 4, p. 825-845, 2008.

BISANG, R.; KATZ, J. Eficacia y eficiencia microeconómica en instituciones no sujetas a reglas convencionales de mercado. Buenos Aires: [s.n.], 1996. Mimeografado.

BONACIM, C. A. G.; ARAUjO, A. M. P. Avaliação de desempenho econômico financeiro dos serviços de saúde: os reflexos das políticas operacionais no setor hospitalar. Ciência \& Saúde Coletiva, v. 16, p. 10551068, 2011.

BRESSER-PEREIRA, L. C. Crise Econômica e Reforma do Estado no Brasil: para uma Nova Interpretação da América Latina.São Paulo: Editora 34, 1996.

BREWER, P. C.; NORREN, E. W.; GARRISON, R.H. Managerial Accounting. 13. ed. New York: McGraw-Hill, 2005.

BRIERS, M.; HIRST, M. The role of budgetary information in performance evaluation. In: EMMANUEL, C.; OTLEY, D.; MERCHANT, K. (Org.) Readings in Accounting for Management Control. London: Chapman \& Hall, 1992.

CARUANA, A.; EWING, M. T.; RAMASESHAN, B. Effects of some environmental challenges and centralization on the entrepreneurial orientation and performance of public sector entities. The Service Industries Journal, v. 22, n. 2, p. 43-58, 2002.

COZZENS, S. E.; MELKERS, J. E. Use and usefulness of performance measurement in state science and technology programs. Policy Studies Journal, v. 25, n. 3, p. 425-435, 1997.

CUNILL GRAU, N.; OSPINA BOZZI, S. La evaluación de los resultados de la gestión pública: una herramienta técnica y política. In: CLAD/ AECI, CUNILL GRAU, N.; OSPINA BOZZI, S. (Org.), Evaluación de Resultados para una Gestión Pública Moderna y Democrática: experiencias latinoamericanas, Caracas, 2003.

ELDENBURG, L. G.; WOLCOTT, S. K. Gestão de Custos: como medir, monitorar e motivar o desempenho. Rio de Janeiro: LTC, 2007.

FIOCRUZ, V Congresso Interno - Relatório
Final da Plenária Extraordinária. Diretrizes para a Adequação da Estrutura Organizacional da Fiocruz, 2006. Disponível em: <http://www.fiocruz.br/ congressointerno/media/relatorio_final_vci_ plenaria.pdf>. Acesso em: 23 mar. 2013.

HARRIS, J. E. The internal organization of hospital: some economic implications. The Bell Journal of Economics, v. 8, n. 2, p. 467-482, 1977.

HASSLOCHER-MORENO, A. M.; JORGE, M. J.; SOUSA, A. S. DE. et al. Atenção integral e eficiência no Laboratório de Pesquisa Clínica em Doenças de Chagas do Instituto de Pesquisa Clínica Evandro Chagas, 2009-2011. Revista Epidemiologia e Serviços de Saúde, v. 22, p. 295-306, 2013.

HORNGREN, C. T.; SUNDEM, G. L.; STRATTOON, W. S. Contabilidade gerencial. 12. ed. São Paulo: Pearson Prentice Hall, 2004.

JOGARATNAM, G.; CHING-YICK TSE, E. Entrepreneurial orientation and the structuring of organizations. International Journal of Contemporary Hospitality Management, v. 18, n. 6, p. 454-468, 2006.

JOHNSON, S.; VAN DE VEN, A. H. A framework for entrepreneurial strategy. In: HIT'T, M. A; IRELAND, R. D; CAMP, S. M.; SEXTON, D. L. (Org.) Strategic entrepreneurship: Creating a new mindset. Oxford, UK: Blackwell Publishers, 2002. p. 66-85.

JORGE, M. J. Mensuração de Performance e Modelo de Gestão Segundo Resultados em Organizações de C\&T do Campo da Saúde: uma Avaliação da Experiência da FIOCRUZ. 2001. 218 f. Tese (Doutorado em Engenharia de Produção)-Universidade Federal do Rio de Janeiro, Rio de Janeiro, 2001.

et al. Custo da Atividade de Assistência, Leitura do Resultado do Exame, Custo da Bancada e Orçamento do Projeto: um Método para a Gestão de Custo da Pesquisa Clínica no IPEC. Rio de Janeiro: IPEC/FIOCRUZ, 2010, mimeo.

et al. Efetividade em custo e análise de eficiência de programas em organizações complexas a experiência do IPEC/FIOCRUZ. Ciudad de México: FUNSALUD, 2006.

LUMPKIN, G. T.; COGLISER, C. C.; SCHNEIDER D. R. Understanding \& measuring autonomy: an entrepreneurial orientation perspective. 
Entrepreneurship Theory and Practice, v. 33, p. 47-69, 2010.

MARTINS, E. Contabilidade de Custos. 9. ed. São Paulo: Editora Atlas, 2008.

MEDICI, A. C.; MARQUES, R. M. Sistemas de custos como instrumento de eficiência e qualidade dos serviços de saúde. Cadernos FUNDAP, n. 19, p. 47-59, jan./abr. 1996.

MINTZBERG, H. Criando Organizações Eficazes, Editora Atlas. São Paulo, 1995.

MORTON, A. N.; HU, Q. Implications of the fit between organizational structure andERP: A structural contingency theory perspective. International Journal of Information Management, v. 28, p. 391402, 2008.

NAKAGAWA, M. ABC: Custeio Baseado em Atividades. São Paulo: Atlas, 1994.

ROBBINS, S. P. Mudança Organizacional e Administração do Estresse. Comportamento Organizacional. 8. ed. Rio de Janeiro: LTC, 1999. p. 394-423.

ROBINSON, R. B. Creating a 21st century entrepreneurial organization. Academy of Entrepreneurship Journal, v. 8, n. 1, p. 321-332, 2007.

ROVERE, M. Políticas y gestión en las organizaciones de ciencia y tecnología en salud. Washington, D.C.: OPAS, 1997. (OPS/HDP/ HDR/97.05). Mimeografado.

SIEGEL, S. Nonparametric Statistics, New York: McGraw-Hill, 1956.

SOUZA, A. A.; GUERRA, M.; LARA, C. O.; GOMIDE, P. L. R.; PEREIRA, C. M.; FREITAS, D.

A. Controle de gestão em organizações hospitalares. Revista de Gestão USP, v. 16, n. 3, p. 15-29, 2009.

SOUZA, M. A.; MAUSS, C. V.; DIEHL, C. A. A gestão pública por resultados e a avaliação de desempenho. In: CONGRESSO USP CONTROLADORIA E CONTABILIDADE, 8., 2008, São Paulo. Anais... São Paulo: USP, 2008.

WARREN, C. S.; REEVE, J. M.; FESS, P. E. Contabilidade gerencial. 2. ed. São Paulo: Thomson Learning, 2008.
YIN, R. K. Estudo de caso: planejamento e métodos. 3. ed. Porto Alegre: Bookman, 2005.

ZAR, J. H. Significance testing of the Spearman Rank Correlation Coefficient. Journal of the American Statistical Association, v. 67, n. 339, p. 578-580, set. 1972. 